\title{
Adaptive variable structure control of a class of non-linear systems
}

\author{
M Ö Efe \\ Electrical and Electronics Engineering, TOBB University of Economics and Technology, Söğütözü Cad No. 43, Ankara \\ 06560, Turkey. email: onderefe@etu.edu.tr
}

The manuscript was received on 3 March 2006 and was accepted after revision for publication on 26 September 2006.

DOI: 10.1243/09596518JSCE257

\begin{abstract}
The problem of tuning the parameters of a controller operating in the presence of noise, time-varying parameters, non-linearities, and uncertainties in practice requires the process of continuous adjustment of the parameters influencing the performance. When the diversity of the challenges in the domain of real-time applications is taken into consideration, it becomes apparent that the adopted controller structure must be sufficiently flexible, and its operating principles must be well explained, particularly in accounting for the stability issues. In this paper, a method is proposed for tuning the parameters of controllers whose outputs are linear in the adjustable parameters, utilizing variable structure systems theory. The method discussed constructs the best error measure at the controller output, and uses this quantity with a suitably designed tuning law, which drives the system under control to a predefined sliding regime and maintains the sliding mode. It is shown analytically that the parametric evolution takes place in a finite volume space, indicating that the controller parameters evolve bounded. In the simulation studies, the approach presented has been tested on the control of a double pendulum system and the superior performance of the strategy has been shown under the existence of noisy observations and large non-zero initial errors.
\end{abstract}

Keywords: sliding mode control, tuning laws, non-linear systems, robust control

\section{INTRODUCTION}

Due to the time-varying and complex nature of real-life systems, synthesizing a control sequence approximating the behaviour of a system under control to that of a reference dynamics is a challenge. The reason that lies behind this is the fact that the available tools of non-linear control theory are not diverse enough to suggest a cost-effective solution to handle these difficulties. Many issues have been addressed through the last few decades of control theory. Particularly the problems related to stability and robustness today constitute the core of any realistic controller design and control sequence synthesis. The mathematical background of the framework is well developed for this task, especially if the model of the system under control is available, or if the extent of the uncertainties is known a priori, or if the statistical models of disturbances are known. However, the practical aspects of the state- of-the-art applications rarely allow the designer to have such information, due to the entirely changing behaviour of the operating environment and the task definitions, which sometimes drives the exploitation of a human expert. A mobile robot navigating in an unknown terrain to fulfil a specific task can be a good example of this challenge. Building a suitable command and control hierarchy for tasks requiring precision without the use of a human expert steers the designer to focus on well-explained tuning strategies, overcoming the inabilities of the traditional methods.

It is a well-known fact that controllers having structural simplicity are easy to implement and are computationally efficient, but the focus of the design is typically confined to the macro dynamics of the system under investigation. On the other hand, controllers taking care of the micro models of the process achieve the goal of precision and autonomy at the cost of performing a multivariable optimization at each step; i.e. the need for costly hardware is of 
interest. Examples of such a case include the use of static or dynamic maps, like neural networks and fuzzy systems, the complexity of which limit the mathematical tractability when accounting for the issues of stability and robustness.

This paper proposes a solution to the problem of synthesizing controllers that are structurally simple and adaptive, and that lead to a robust and highperformance control system by adopting the variable structure systems (VSS) theory. VSS theory is well known, with its robustness to uncertainties and disturbances. Conceptually, the controller design in VSS is based on the nominal representation of the system about which the bounds of the uncertainties are assumed to be available. The decision mechanism operates on the basis of switching on the different sides of a decision boundary, which is called the sliding hypersurface [1-3], and the aim of the design is to enforce the error vector towards this hypersurface during the reaching phase. Once the error vector is confined to the sliding hypersurface, it obeys the behaviour imposed by the set of equations describing the hypersurface, i.e. the sliding mode starts and the error vector converges to the origin. The control strategy is therefore called sliding mode control (SMC) in the related literature [1-3]. During the sliding mode, the control system becomes totally insensitive to the disturbances and uncertainties unless the decision mechanism violates the physical limits for maintaining the sliding motion.

The SMC strategy has been applied successfully in a wide variety of design problems, ranging from the control of chemical processes [4-7] to the control of chaotic systems [8]. Hung et al. [1] review the control strategy for linear and non-linear systems and discuss the design for systems represented in canonical forms. Another systematic examination of the SMC approach is presented in reference [9], in which the practical aspects of SMC design are assessed for both continuous-time and discrete-time cases and a special consideration is given to the finite switching frequency, limited bandwidth actuators, and parasitic dynamics. Studies considering the reparameterization of the sliding surface constitute an important branch of introducing adaptation into the control system [10]. Depending on the observed response, the sliding surface evolves in time such that the error vector becomes trapped in the sliding manifold in a short time and converges to the origin afterwards. Misawa discusses the SMC task for discrete-time systems in reference [11] for the linear case and in reference [12] for the non-linear case with unmatched uncertainties, Sabanovic et al. [13] elaborate on the concept of chattering-free SMC design, Bartolini et al. [14] formulate the chatteringfree SMC for multi-input-multi-output (MIMO) systems, and Erbatur et al. [15] investigate the robustness properties of the SMC technique on a two-degree-of-freedom (2-DOF) direct-drive SCARA (selective compliant articulated robot in assembly).

Studies demonstrating the high performance of the SMC scheme in handling the uncertainties and imprecision have motivated the use of the SMC scheme in tuning the parameters of flexible systems $[\mathbf{1 6}, \mathbf{1 7}]$. The methods discussed in these references deal with the dynamic adaptation of the parameters of a flexible model such that the error on the output of the model tends to zero in finite time. The first results, discussed by Sira-Ramirez and Colina-Morles [16] are on the inverse dynamics identification of a Kapitsa pendulum by using constant bounds for the uncertainties. Yu et al. [17] extend the results of reference [16] by introducing adaptive uncertainty bound dynamics, and their work focuses on the same example. If the flexible structure is to be used as a controller, the dynamic adaptation mechanism in references [16] and [17] needs the error on the output of the controller. This fact constitutes a major difficulty because error on the applied control signal is unavailable. Efe et al. [18] consider the problem of constructing the equivalent measure of the error on the controller output in a qualitative manner. More explicitly, a relation between the sliding surface equation and the control error is proposed and the conditions that the relation must satisfy are discussed; the results are assessed on the dynamic model of a 3-DOF anthropoid robotic manipulator. In reference [19], a dynamic learning scheme based on SMC is discussed for the training of feedforward neural networks. The method has been used for the identification of a periodic time signal. The potential difficulty in implementing the algorithm is the fact that the hidden neuron outputs are differentiated with respect to time for evaluating the value of the switching function. Although this difficulty can somehow be alleviated through filtering techniques, the problem of the control error unavailability restricts the applicability in terms of the needs of control engineering expertise.

The focus of this paper is to use the tuning algorithm proposed by Sira-Ramirez and ColinaMorles [16] for tuning the parameters of a simple controller driving the plant under control to the predefined sliding regime and maintaining the sliding mode. The primary difference from what is presented in this paper and what is considered in reference [18] 
is the form of the equivalent control error, which is postulated through the concept of a reference SMC model. In the second section, the conventional SMC approach is briefly introduced. The details of what is novel are presented in the third section. The section gives the formulation, requirements, and relevant stability analyses and discusses several issues related to the practice. Furthermore, it is proved in the third section that the parameters of the controller evolve bounded as time progresses. In the fourth section, a case study considering the control of the double pendulum system is presented. The conclusions constitute the last part of the paper.

\section{CONVENTIONAL SLIDING MODE CONTROL DESIGN REVISITED}

Design of a controller for a system, whose salient features are represented in the model upon which the design is based, requires the alleviation of plant/model mismatches. These mismatches can be classified into two categories, namely structured and unstructured. In the former, uncertainties are on the values of the parameters, while the latter concerns the unmodelled dynamics existing in the plant [3]. For the structured uncertainties, the framework of adaptive control offers well-formulated solutions based on the estimation of slowly varying unknown parameters. The design of the controller can then be realized using the estimates. However, an oversimplified or an imperfect model of the plant necessitates a very robust controller to maintain the tracking performance. Use of VSS theory is one particular approach for achieving this task [20].

Consider a non-linear and non-autonomous system represented as

$$
\theta_{i}^{\left(r_{i}\right)}=f_{\mathrm{p}_{i}}(\boldsymbol{\theta})+\sum_{j=1}^{n} d_{i j} \tau_{j}, \quad i=1, \ldots, n
$$

The system under control is a multi-input-multioutput one driven by the $n \times 1$-dimensional input vector $\tau$. The state vector and the input vector of the system in equation (1) are defined as

$$
\begin{aligned}
\boldsymbol{\theta} & =\left[\begin{array}{lllllllll}
\theta_{1} & \dot{\theta}_{1} & \cdots & \theta_{1}^{\left(r_{1}-1\right)} & \cdots & \theta_{n} & \dot{\theta}_{n} & \cdots & \theta_{n}^{\left(r_{n}-1\right)}
\end{array}\right]^{\mathrm{T}} \\
\boldsymbol{\tau} & =\left[\begin{array}{llll}
\tau_{1} & \tau_{2} & \cdots & \tau_{n}
\end{array}\right]^{\mathrm{T}}
\end{aligned}
$$

respectively. The system in equation (1) can be rewritten as

$$
\dot{\boldsymbol{\theta}}=\boldsymbol{f}_{\mathrm{p}}(\boldsymbol{\theta})+\mathrm{D} \boldsymbol{\tau}
$$

in which $\mathbf{D}$ denotes the input gain matrix. Without loss of generality, the vector of sliding surfaces is chosen as a linear function of the components of the tracking error vector described as

$$
\begin{aligned}
& \boldsymbol{e}=\left[\begin{array}{lllllllll}
e_{1} & \dot{e}_{1} & \cdots & e_{1}^{\left(r_{1}-1\right)} & \cdots & e_{n} & \dot{e}_{n} & \cdots & e_{n}^{\left(r_{n}-1\right)}
\end{array}\right]^{\mathrm{T}}
\end{aligned}
$$

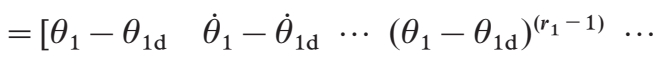

$$
\begin{aligned}
& \left.\theta_{n}-\theta_{n \mathrm{~d}} \quad \dot{\theta}_{n}-\dot{\theta}_{n \mathrm{~d}} \cdots\left(\theta_{n}-\theta_{n \mathrm{~d}}\right)^{\left(r_{n}-1\right)}\right]^{\mathrm{T}}
\end{aligned}
$$

which is the discrepancy between the values of the measured state vector $\boldsymbol{\theta}$ and the desired state vector $\boldsymbol{\theta}_{\mathrm{d}}$. The sliding surface $\boldsymbol{s}_{\mathrm{p}}(\boldsymbol{e})$ is an $n \times 1$ vector and is defined as

$$
s_{\mathrm{p}}(\boldsymbol{e})=\mathbf{G} \boldsymbol{e}=\mathbf{G}\left(\boldsymbol{\theta}-\boldsymbol{\theta}_{\mathrm{d}}\right)
$$

The widespread selection of the matrix $\mathbf{G}$ is such that the $i$ th sliding surface function has the form

$$
s_{\mathrm{p}_{i}}\left(e_{i}\right)=\left(\frac{\mathrm{d}}{\mathrm{d} t}+\lambda_{i}\right)^{r_{i}-1} e_{i}
$$

in which $\lambda_{i}$ is a strictly positive constant. Let $V_{\mathrm{p}}$ be a candidate Lyapunov function given as

$$
V_{\mathrm{p}}\left(\boldsymbol{s}_{\mathrm{p}}\right)=\frac{1}{2} \boldsymbol{s}_{\mathrm{p}}^{\mathrm{T}} \boldsymbol{s}_{\mathrm{p}}
$$

If the prescribed control signal satisfies

$$
\dot{V}_{\mathrm{p}}\left(s_{\mathrm{p}}\right)=-s_{\mathrm{p}}^{\mathrm{T}} \xi \operatorname{sgn}\left(s_{\mathrm{p}}\right)
$$

the negative definiteness of the time derivative of the Lyapunov function in equation (8) is ensured. In equation (9), $\xi$ is a positive definite diagonal matrix of dimension $n \times n$. If the time derivative of the Lyapunov function in equation (8) is evaluated, the quantity obtained must be equal to the expression in equation (9). This can be stated as

$$
\boldsymbol{s}_{\mathrm{p}}^{\mathrm{T}} \dot{s}_{\mathrm{p}}=-\boldsymbol{s}_{\mathrm{p}}^{\mathrm{T}} \xi \operatorname{sgn}\left(\boldsymbol{s}_{\mathrm{p}}\right)
$$

From equations (4) and (6), the time derivative for $\boldsymbol{s}_{\mathrm{p}}$ can be written as

$$
\dot{s}_{\mathrm{p}}=-\mathbf{G} \dot{\boldsymbol{\theta}}_{\mathrm{d}}+\mathbf{G}\left(f_{\mathrm{p}}(\boldsymbol{\theta})+\mathbf{D} \boldsymbol{\tau}\right)
$$

Substituting equation (11) into equation (10), the control signal can be constructed as

$$
\tau=\tau_{\mathrm{eq}}+\boldsymbol{\tau}_{\mathrm{c}}
$$

in which the first term is the equivalent control term and the second term is the corrective control term. Both terms are explicitly given as in equations (13) and (14) respectively. For the existence of the mentioned components, the matrix GD must not be 
rank deficient

$$
\begin{aligned}
& \boldsymbol{\tau}_{\mathrm{eq}}=-(\mathbf{G D})^{-1}\left[\mathbf{G} \boldsymbol{f}_{\mathrm{p}}(\boldsymbol{\theta})-\mathbf{G} \dot{\boldsymbol{\theta}}_{\mathrm{d}}\right] \\
& \boldsymbol{\tau}_{\mathrm{c}}=-(\mathbf{G D})^{-1} \boldsymbol{\xi} \operatorname{sgn}\left(\boldsymbol{s}_{\mathrm{p}}\right)
\end{aligned}
$$

In the literature, equivalent control is considered as the low-frequency (average) component of the control signal. Because of the discontinuity on the sliding surface, the corrective term introduces the switching component [2, 21].

If $\boldsymbol{e}(0)=\boldsymbol{O}$, the tracking problem can be considered as keeping $\boldsymbol{e}$ on the sliding surface, but, for non-zero initial conditions, the strategy must enforce the state trajectories towards the sliding surface, which is ensured by the negative definiteness of the time derivative of the Lyapunov function as in equation (9). For the case of non-zero initial conditions, the phase until the error vector hits the sliding surface is called the reaching mode, the dynamic characteristics of the system during which the control strategy to be adopted is determined. Application of the control input formulated in equation (12) imposes the dynamics described as

$$
\dot{s}_{\mathrm{p}}=-\xi \operatorname{sgn}\left(s_{\mathrm{p}}\right)
$$

which clearly enforce the error vector towards the sliding surface. Once the sliding surface is reached, the value of equation (7) becomes zero, and this enforces the error vector to move towards the origin.

Lastly in this section, it is beneficial to mention the problems associated with the SMC strategy in devising variable structure controllers. The first problem stems from the discontinuity of the control signal about the sliding surface. After the reaching phase, the constructed form of the control signal enforces the system states to lie on the sliding surface, along which a discontinuous control action is of interest. This fact introduces high-frequency components into the prescribed form of the control signal, the application of which may excite the undesired high-frequency dynamics of the plant under control and can lead to unpredictable instabilities. The problem of having such high-frequency components in the control signal is referred to as chattering in the literature $[\mathbf{1}, \mathbf{9}, \mathbf{2 0}]$. For the alleviation of the chattering phenomenon, various techniques have been reported in the literature, which postulate the form of the control signal for the plants, whose governing equations are linear in the control term $[13,15]$.

If the observed state variables are noisy, the control signal is adversely affected by this parasitic dynamics. Since the form of control signal entails the sign of a measured quantity, which is very close to zero, the problem can be solved by introducing a thin boundary layer, in which the control signal is smoothed out [3].

\section{DEFINITIONS AND THE FORMULATION OF THE PARAMETER TUNING STRATEGY}

\subsection{Constructing a measure of the equivalent control error}

\section{Remark 1}

The SMC task is achievable if the dynamics of the system in equation (1) is totally known or if the nominal system is known with the bounds of the uncertainties. It must be noted that to satisfy the matching conditions, the disturbances and uncertainties are always assumed to enter the system through the control channels [1]. When the conventional SMC strategy is applied to the system of equation (1), the resulting behaviour is called the target SMC and the input vector leading to it the target control sequence $(\tau)$, which is described in equations (12) to (14). If the functional form of the vector function $\boldsymbol{f}_{\mathrm{p}}$ is not known, it should be obvious that the target control sequence cannot be constructed by following the traditional SMC design approaches.

\section{Definition 1}

Given an uncertain plant, which has the structure described as in equation (1), and a command trajectory vector $\boldsymbol{\theta}_{\mathrm{d}}(t)$ for $t \geqslant 0$, the input sequence satisfying the following vector differential equation is defined to be the idealized control sequence, denoted by $\tau_{\mathrm{d}}$, and the vector differential equation itself is defined to be the reference SMC model

$$
\dot{\boldsymbol{\theta}}_{\mathrm{d}}=\boldsymbol{f}_{\mathrm{p}}\left(\boldsymbol{\theta}_{\mathrm{d}}\right)=\mathrm{D} \tau_{\mathrm{d}}
$$

Mathematically, the existence of such a model and the sequence means that the system of equation (1) follows the command trajectory vector perfectly if both the idealized control sequence is known and the initial conditions are set as $\boldsymbol{\theta}(t=0)=\boldsymbol{\theta}_{\mathrm{d}}(t=0)$, more explicitly $\boldsymbol{e}(t) \equiv 0$ for $\forall t \geqslant 0$. Undoubtedly, such an idealized control sequence will not be a normbounded signal when there are step-like changes in the vector of command trajectories or when the initial errors are non-zero. Therefore the reference SMC model is an abstraction due to the limitations imposed by the physical reality, but the concept of an idealized control sequence should be viewed as 
the synthesis of the command signal $\boldsymbol{\theta}_{\mathrm{d}}$ from the time solution of the differential equation set in equation (16).

\section{Fact 1}

Based on the Lyapunov stability results of the previous section, if the target control sequence formulated in equation (12) is applied to the system of equation (1), the idealized control sequence would be the steady state solution of the control signal, i.e. $\lim _{t \rightarrow \infty} \tau=\tau_{\mathrm{d}}$. However, under the assumption of the achievability of the SMC task, the difficulty here is again the unavailability of the functional form of the vector function $\boldsymbol{f}_{\mathrm{p}}$. Therefore, the aim in this subsection is to discover an equivalent form of the discrepancy between the control applied to the system and its target value by utilizing the idealized control viewpoint. This discrepancy measure is denoted by $\boldsymbol{s}_{\mathrm{c}}=\boldsymbol{\tau}-\boldsymbol{\tau}_{\mathrm{d}}$ and is of dimension $n \times 1$. If the target control sequence of equation (12) is rewritten using equation (16), then

$$
\begin{aligned}
\boldsymbol{\tau} & =-(\mathbf{G D})^{-1}\left[\mathbf{G} \boldsymbol{f}_{\mathrm{p}}(\boldsymbol{\theta})-\mathbf{G}\left(\boldsymbol{f}_{\mathrm{p}}\left(\boldsymbol{\theta}_{\mathrm{d}}\right)+\mathbf{D} \boldsymbol{\tau}_{\mathrm{d}}\right)+\xi \operatorname{sgn}\left(\boldsymbol{s}_{\mathrm{p}}\right)\right] \\
& =-(\mathbf{G D})^{-1}\left[\mathbf{G} \boldsymbol{f}_{\mathrm{p}}(\boldsymbol{\theta})-\mathbf{G} \boldsymbol{f}_{\mathrm{p}}\left(\boldsymbol{\theta}_{\mathrm{d}}\right)+\xi \operatorname{sgn}\left(\boldsymbol{s}_{\mathrm{p}}\right)\right]+\boldsymbol{\tau}_{\mathrm{d}} \\
& =-(\mathbf{G D})^{-1}\left[\mathbf{G} \boldsymbol{\Delta} \boldsymbol{f}_{\mathrm{p}}(\boldsymbol{\theta})+\xi \operatorname{sgn}\left(\boldsymbol{s}_{\mathrm{p}}\right)\right]+\boldsymbol{\tau}_{\mathrm{d}}
\end{aligned}
$$

The target control sequence becomes identical to the idealized control sequence, i.e. $\tau \equiv \tau_{\mathrm{d}}$, as long as the condition in equation (18) holds true. However, this condition is of no practical importance as the analytic form of the vector function $\boldsymbol{f}_{\mathrm{p}}$ is not known. Therefore, this equality should be considered as an equality to be enforced instead of an equality that holds true all the time, because its implication is $\boldsymbol{s}_{\mathrm{c}}=0$ and is the aim of the design

$$
\mathbf{G} \Delta \boldsymbol{f}_{\mathrm{p}}(\boldsymbol{\theta})+\boldsymbol{\xi} \operatorname{sgn}\left(\boldsymbol{s}_{\mathrm{p}}\right)=0
$$

It is obvious that to enforce the equality in equation (18) the target control sequence should be synthesized, which will ultimately converge to the idealized control sequence by the adaptation algorithm yet to be discussed.
Consider the time derivative of the vector of sliding surfaces

$$
\begin{aligned}
\dot{\boldsymbol{s}}_{\mathrm{p}}(\boldsymbol{e}) & =\mathbf{G} \dot{\boldsymbol{e}} \\
& =\mathbf{G}\left(\dot{\boldsymbol{\theta}}-\dot{\boldsymbol{\theta}}_{\mathrm{d}}\right) \\
& =\mathbf{G}\left(\boldsymbol{f}_{\mathrm{p}}(\boldsymbol{\theta})+\mathbf{D} \boldsymbol{\tau}-\boldsymbol{f}_{\mathrm{p}}\left(\boldsymbol{\theta}_{\mathrm{d}}\right)-\mathbf{D} \boldsymbol{\tau}_{\mathrm{d}}\right) \\
& =\mathbf{G}\left(\Delta f_{\mathrm{p}}+\mathbf{D}\left(\boldsymbol{\tau}-\boldsymbol{\tau}_{\mathrm{d}}\right)\right) \\
& =\mathbf{G}\left(\Delta f_{\mathrm{p}}+\mathbf{D} s_{\mathrm{c}}\right)
\end{aligned}
$$

Utilizing the relation (18) in equation (19) and solving for $\boldsymbol{s}_{\mathrm{c}}$ yields the following relation

$$
s_{\mathrm{c}}=(\mathbf{G D})^{-1}\left[\dot{s}_{\mathrm{p}}+\xi \operatorname{sgn}\left(s_{\mathrm{p}}\right)\right]=\boldsymbol{\tau}-\boldsymbol{\tau}_{\mathrm{d}}
$$

\section{Remark 2}

The reader must here notice that the application of $\tau_{\mathrm{d}}$ to the system of equation (1) with zero initial errors will lead to $\boldsymbol{e}(t) \equiv 0$ for $\forall t \geqslant 0$; on the other hand, the application of $\tau$ to the system of equation (1) will lead to $\boldsymbol{s}_{\mathrm{p}}=\boldsymbol{O}$ for $\forall t \geqslant t_{\mathrm{h}}$, where $t_{\mathrm{h}}$ is the hitting time, and the origin will be reached according to the dynamics of the sliding manifold. Therefore, the adoption of equation (20) as the equivalent measure of the control error loosens the $\boldsymbol{e}(t) \equiv 0$ for $\forall t \geqslant 0$ requirement and induces all trajectories in the error space to tend to the sliding hypersurface, i.e. equation (15) is enforced. Consequently, the tendency of the control scheme will be to generate the target SMC sequence of equation (12).

Now consider the ordinary feedback control loop illustrated in Fig. 1 and define the following Lyapunov function, which is a measure of how well the controller performs

$$
V_{\mathrm{c}}\left(s_{\mathrm{c}}\right)=\frac{1}{2} s_{\mathrm{c}}^{\mathrm{T}} s_{\mathrm{c}}
$$

\section{Remark 3}

An adaptation algorithm ensuring $\dot{V}_{\mathrm{c}}\left(\boldsymbol{s}_{\mathrm{c}}\right)<0$ when $\boldsymbol{s}_{\mathrm{c}} \neq 0$ enforces $\mathbf{G} \boldsymbol{\Delta} \boldsymbol{f}_{\mathrm{p}}(\boldsymbol{\theta})+\boldsymbol{\xi} \operatorname{sgn}\left(\boldsymbol{s}_{\mathrm{p}}\right)=0$ and creates the predefined sliding regime after a reaching mode

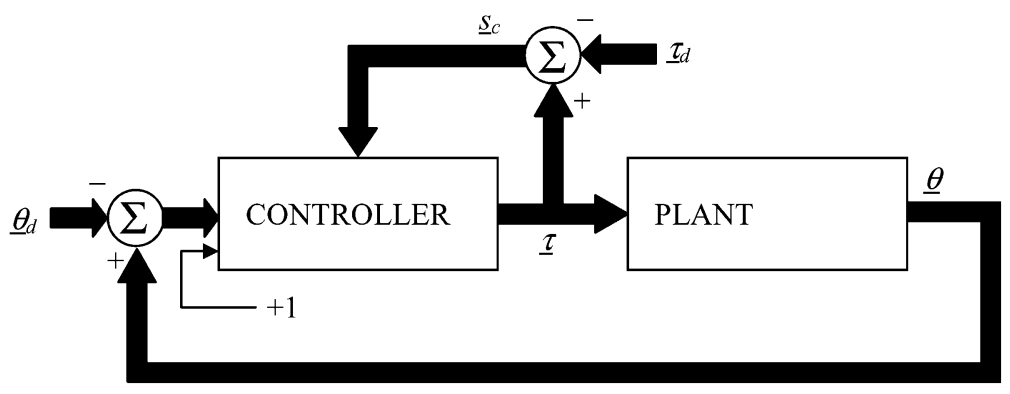

Fig. 1 Block diagram of the control system 
lasting until the hitting time denoted by $t_{\mathrm{h}}$, beyond which $\boldsymbol{s}_{\mathrm{c}}=\boldsymbol{O}$ as the system is in the sliding regime.

$$
\begin{aligned}
& \text { If } \dot{V}_{\mathrm{c}}\left(\boldsymbol{s}_{\mathrm{c}}\right)<0 \text { when } \boldsymbol{s}_{\mathrm{c}} \neq 0 \text {, then } \\
& \lim _{t \rightarrow t_{\mathrm{h}}} V_{\mathrm{c}}=0 \Leftrightarrow \lim _{t \rightarrow t_{\mathrm{h}}}\left\|\boldsymbol{s}_{\mathrm{c}}\right\|=0 \Leftrightarrow \lim _{t \rightarrow t_{\mathrm{h}}}\left\|\dot{\boldsymbol{s}}_{\mathrm{p}}+\xi \operatorname{sgn}\left(\boldsymbol{s}_{\mathrm{p}}\right)\right\|=0
\end{aligned}
$$

Note that the meaning of $\boldsymbol{s}_{\mathrm{c}}=\boldsymbol{O}$ is now equivalent to $\boldsymbol{s}_{\mathrm{p}}=\boldsymbol{O}$ by Remark 2 . Therefore the limits above are evaluated as $t \rightarrow t_{\mathrm{h}}$.

\subsection{Structure of the controller}

Consider the non-linear non-autonomous system described in equation (1). For the $i$ th subsystem, define the controller input vector as

$$
\boldsymbol{u}_{i}=\left[\begin{array}{lllll}
e_{i} & \dot{e}_{i} & \cdots & e_{i}^{\left(r_{i}-1\right)} & 1
\end{array}\right]^{\mathrm{T}}
$$

and define the adjustable parameter vector as

$$
\boldsymbol{\phi}_{i}=\left[\begin{array}{lllll}
\phi_{i 1} & \phi_{i 2} & \cdots & \phi_{i, r_{i}} & \phi_{i, r_{i}+1}
\end{array}\right]^{\mathrm{T}}
$$

The input-output relation of the controller is described as

$$
\tau_{i}=\boldsymbol{\phi}_{i}^{\mathrm{T}} \boldsymbol{u}_{i}
$$

\subsection{Adaptation algorithm}

In order not to be in conflict with the physical reality, the designer must impose the following inequalities, the truth of which state that the parameters of the controller, the time derivative of the signal exciting the controller, and the time derivative of the idealized output of the controller remain bounded

$$
\begin{gathered}
\left\|\boldsymbol{\phi}_{i}\right\| \leqslant B_{\phi_{i}} \\
\left\|\dot{\boldsymbol{u}}_{i}\right\| \leqslant B_{\dot{u}_{i}} \\
\left\|\dot{\tau}_{i \mathrm{~d}}\right\| \leqslant B_{\dot{i}_{i \mathrm{~d}}}
\end{gathered}
$$

Note that in Definition 1, it is stated that there may not be a finite $B_{i_{i \mathrm{~d}}} \in \Re$, even in some realistic situations like non-zero initial errors. However, the practical meaning of imposing (27) leads to the construction of an approximation of the idealized control sequence and the requirement of $\boldsymbol{e}(t) \equiv 0$ for $\forall t \geqslant 0$ must therefore be loosened.

\section{Theorem 1}

For the $i$ th subsystem of the system described in equation (1), adopting the controller of structure (24), the adaptation of controller parameters as described in the following equation enforces the value of the $i$ th component of the control discrepancy vector $\left(\boldsymbol{s}_{\mathrm{c}_{i}}\right)$ to zero

$$
\dot{\boldsymbol{\phi}}_{i}=-\frac{\boldsymbol{u}_{i}}{\boldsymbol{u}_{i}^{\mathrm{T}} \boldsymbol{u}_{i}} k_{i} \operatorname{sgn}\left(\boldsymbol{s}_{\mathrm{c}_{i}}\right)
$$

where $k_{\mathrm{i}}$ is a sufficiently large positive constant satisfying

$$
k_{i}>B_{\phi_{i}} B_{\dot{u}_{i}}+B_{\dot{\tau}_{i \mathrm{~d}}}
$$

The adaptation mechanism in equation (28) drives an arbitrary initial value of $\boldsymbol{s}_{\mathrm{c}_{i}}$ to zero in finite time, denoted by $t_{\mathrm{h}_{i}}$ satisfying the inequality in

$$
t_{\mathrm{h}_{i}} \leqslant \frac{\left|\mathbf{s}_{\mathrm{c}_{i}}(0)\right|}{k_{i}-\left(B_{\phi_{i}} B_{\dot{u}_{i}}+B_{\dot{\tau}_{\mathrm{id}}}\right)}
$$

Proof. Consider the Lyapunov function candidate in

$$
V_{\mathrm{c}_{i}}=\frac{1}{2} s_{\mathrm{c}_{i}}^{2}
$$

In order to reach the zero error level $\left(s_{\mathrm{c}_{i}}=0\right)$, the time derivative of equation (31) must be negative definite, which is given in

$$
\begin{aligned}
\dot{V}_{\mathrm{c}_{i}} & =\dot{s}_{\mathrm{c}_{i}} s_{\mathrm{c}_{i}} \\
& =\left(\dot{\tau}_{i}-\dot{\tau}_{i \mathrm{~d}}\right) s_{\mathrm{c}_{i}} \\
& =\left(\dot{\boldsymbol{\phi}}_{i}^{\mathrm{T}} \boldsymbol{u}_{i}+\boldsymbol{\phi}_{i}^{\mathrm{T}} \dot{\boldsymbol{u}}_{i}-\dot{\tau}_{i \mathrm{~d}}\right) s_{\mathrm{c}_{i}} \\
& =-k_{i} \operatorname{sgn}\left(s_{\mathrm{c}_{i}}\right) s_{\mathrm{c}_{i}}+\left(\boldsymbol{\phi}_{i}^{\mathrm{T}} \dot{\boldsymbol{u}}_{i}-\dot{\tau}_{i \mathrm{~d}}\right) s_{\mathrm{c}_{i}} \\
& =-k_{i}\left|s_{\mathrm{c}_{i}}\right|+\left(\boldsymbol{\phi}_{i}^{\mathrm{T}} \dot{\boldsymbol{u}}_{i}-\dot{\tau}_{i \mathrm{~d}}\right) s_{\mathrm{c}_{c_{i}}} \\
& <\left(-k_{i}+B_{\phi_{i}} B_{\dot{u}_{i}}+B_{\dot{i}_{i \mathrm{~d}}}\right)\left|s_{\mathrm{c}_{i}}\right|
\end{aligned}
$$

It is apparent that the condition in equation (29) ensures the negative definiteness of the time derivative of the selected Lyapunov function.

If $\dot{s}_{\mathrm{c}}$ is evaluated with the aid of equation (28), the expression in the following equation is obtained

$$
\dot{s}_{\mathrm{c}_{i}}=-k_{i} \operatorname{sgn}\left(s_{\mathrm{c}_{i}}\right)+\boldsymbol{\phi}_{i}^{\mathrm{T}} \dot{\boldsymbol{u}}_{i}-\dot{\tau}_{i \mathrm{~d}}
$$

The solution to the differential equation in (33) can be given by

$$
\begin{aligned}
s_{\mathrm{c}_{i}}(t)-s_{\mathrm{c}_{i}}(0)= & -k_{i} t \operatorname{sgn}\left[s_{\mathrm{c}_{i}}(0)\right] \\
& +\int_{0}^{t}\left[\boldsymbol{\phi}_{i}^{\mathrm{T}}(\sigma) \dot{\boldsymbol{u}}_{i}(\sigma)-\dot{t}_{i \mathrm{~d}}(\sigma)\right] \mathrm{d} \sigma
\end{aligned}
$$

$$
\begin{aligned}
& \text { At } t=t_{\mathrm{h} i}, s_{\mathrm{c}}\left(t_{\mathrm{h} i}\right)=0 \\
& -s_{\mathrm{c}_{i}}(0)=-k_{i} t_{\mathrm{h}_{i}} \operatorname{sgn}\left[s_{\mathrm{c}_{i}}(0)\right] \\
& +\int_{0}^{t_{\mathrm{h}_{i}}}\left[\boldsymbol{\phi}_{i}^{\mathrm{T}}(\boldsymbol{\sigma}) \dot{\boldsymbol{u}}_{i}(\sigma)-\dot{\tau}_{i \mathrm{~d}}(\sigma)\right] \mathrm{d} \sigma
\end{aligned}
$$

By multiplying both sides of equation (35) by $-\operatorname{sgn}\left[s_{c_{i}}(0)\right]$,

$$
\begin{aligned}
& \left|s_{\mathrm{c}_{i}}(0)\right|=k_{i} t_{\mathrm{h}_{i}} \\
& -\left\{\int_{0}^{t_{\mathrm{h}_{i}}}\left[\boldsymbol{\phi}_{i}^{\mathrm{T}}(\sigma) \dot{\boldsymbol{u}}_{i}(\sigma)-\dot{i}_{i \mathrm{~d}}(\sigma)\right] \mathrm{d} \sigma\right\} \operatorname{sgn}\left[s_{\mathrm{c}_{i}}(0)\right] \\
& \geqslant k_{i} t_{\mathrm{h}_{i}}-\left(B_{\phi_{i}} B_{\dot{u}_{i}}+B_{i_{i \mathrm{~d}}}\right) t_{\mathrm{h}_{i}}
\end{aligned}
$$


which implies hitting in finite time, as described by the inequality in equation (30).

Note that the assumptions in equations (25) to (27) describe the conditions under which the closed-loop control system behaves in a stable fashion. In other words, the algorithm with the mentioned conditions ensures local stability.

\section{Theorem 2}

If the system enters the sliding mode $s_{\mathrm{c}}=0$ and remains in it thereafter, then the parameters of the $i$ th flexible controller, $\phi_{i}$, evolve in a bounded manner.

Proof. In the sliding mode, $s_{\mathrm{c}_{i}}=0$ and $\dot{s}_{\mathrm{c}_{i}}=0$. Based on this, the following derivation can be made

$$
\begin{aligned}
\dot{s}_{\mathrm{c}_{i}}= & \dot{\tau}_{i}-\dot{\tau}_{i \mathrm{~d}} \\
\dot{s}_{\mathrm{c}_{i}} & =\dot{\boldsymbol{\phi}}_{i}^{\mathrm{T}} \boldsymbol{u}_{i}+\boldsymbol{\phi}_{i}^{\mathrm{T}} \dot{\boldsymbol{u}}_{i}-\dot{\tau}_{i \mathrm{~d}}=0 \\
\boldsymbol{u}_{i}^{\mathrm{T}} \dot{\boldsymbol{\phi}}_{i} & =-\dot{\boldsymbol{u}}_{i}^{\mathrm{T}} \boldsymbol{\phi}_{i}+\dot{\tau}_{i \mathrm{~d}} \\
& =-\frac{\boldsymbol{u}_{i}^{\mathrm{T}} \boldsymbol{u}_{i}}{\boldsymbol{u}_{i}^{\mathrm{T}} \boldsymbol{u}_{i}} \dot{\boldsymbol{u}}_{i}^{\mathrm{T}} \boldsymbol{\phi}_{i}+\frac{\boldsymbol{u}_{i}^{\mathrm{T}} \boldsymbol{u}_{i}}{\boldsymbol{u}_{i}^{\mathrm{T}} \boldsymbol{u}_{i}} \dot{\tau}_{\mathrm{d}} \\
& =\boldsymbol{u}_{i}^{\mathrm{T}}\left(-\frac{\boldsymbol{u}_{i}}{\boldsymbol{u}_{i}^{\mathrm{T}} \boldsymbol{u}_{i}} \dot{\boldsymbol{u}}_{i}^{\mathrm{T}} \boldsymbol{\phi}_{i}+\frac{\boldsymbol{u}_{i}}{\boldsymbol{u}_{i}^{\mathrm{T}} \boldsymbol{u}_{i}} \dot{\tau}_{i \mathrm{~d}}\right)
\end{aligned}
$$

which requires

$$
\boldsymbol{u}_{i}^{\mathrm{T}}\left(\dot{\boldsymbol{\phi}}_{i}+\frac{\boldsymbol{u}_{i}}{\boldsymbol{u}_{i}^{\mathrm{T}} \boldsymbol{u}_{i}} \dot{\boldsymbol{u}}_{i}^{\mathrm{T}} \boldsymbol{\phi}_{i}-\frac{\boldsymbol{u}_{i}}{\boldsymbol{u}_{i}^{\mathrm{T}} \boldsymbol{u}_{i}} \dot{\tau}_{\mathrm{id}}\right)=0
$$

Since the entries of the vector $\boldsymbol{u}_{i}$ cannot be linearly dependent for all time [3], the equality in equation (40) imposes the following differential equation form in the sliding mode

$$
\dot{\boldsymbol{\phi}}=-\frac{\boldsymbol{u}_{i} \dot{\boldsymbol{u}}_{i}^{\mathrm{T}}}{\boldsymbol{u}_{i}^{\mathrm{T}} \boldsymbol{u}_{i}} \phi_{i}+\frac{\boldsymbol{u}_{i}}{\boldsymbol{u}_{i}^{\mathrm{T}} \boldsymbol{u}_{i}} \dot{\tau}_{i \mathrm{~d}}
$$

The solution to the above equation is

$$
\boldsymbol{\phi}_{i}(t)=\boldsymbol{\Phi}_{i}(t, 0) \phi_{i}(0)+\int_{0}^{t} \boldsymbol{\Phi}_{i}(t, \sigma) \frac{\boldsymbol{u}_{i}(\sigma)}{\boldsymbol{u}_{i}(\sigma)^{\mathrm{T}} \boldsymbol{u}_{i}(\sigma)} \dot{\tau}_{i \mathrm{~d}}(\sigma) \mathrm{d} \sigma
$$

where

$$
\boldsymbol{\Phi}_{i}\left(t, t_{0}\right)=\exp \left[-\int_{t_{0}}^{t} \frac{\boldsymbol{u}_{i}(\sigma) \dot{\boldsymbol{u}}_{i}(\sigma)^{\mathrm{T}}}{\boldsymbol{u}_{i}(\sigma)^{\mathrm{T}} \boldsymbol{u}_{i}(\sigma)} \mathrm{d} \sigma\right]
$$

Since $1 \leqslant\left\|\boldsymbol{u}_{i}\right\| \leqslant B_{u_{i}}$, for the first term in equation (42), the following relations can be induced

$$
\begin{aligned}
\left\|\boldsymbol{\Phi}_{i}(t, 0)\right\| & =\left\|\exp \left[-\int_{0}^{t} \frac{\boldsymbol{u}_{i}(\sigma) \dot{\boldsymbol{u}}_{i}(\sigma)^{\mathrm{T}}}{\boldsymbol{u}_{i}(\sigma)^{\mathrm{T}} \boldsymbol{u}_{i}(\sigma)} \mathrm{d} \sigma\right]\right\| \\
& =\left\|\exp \left[-\int_{0}^{t} \frac{\boldsymbol{u}_{i}(\sigma)}{\boldsymbol{u}_{i}(\sigma)^{\mathrm{T}} \boldsymbol{u}_{i}(\sigma)} \mathrm{d} \boldsymbol{u}_{i}(\sigma)^{\mathrm{T}}\right]\right\| \\
& \leqslant\left\|\exp \left[\left|-\int_{0}^{t} \frac{\boldsymbol{u}_{i}(\sigma)}{\boldsymbol{u}_{i}(\sigma)^{\mathrm{T}} \boldsymbol{u}_{i}(\sigma)} \mathrm{d} \boldsymbol{u}_{i}(\sigma)^{\mathrm{T}}\right|\right]\right\| \\
& \leqslant\left\|\exp \left[\int_{0}^{t} \frac{\left|\boldsymbol{u}_{i}(\sigma)\right|}{\boldsymbol{u}_{i}(\sigma)^{\mathrm{T}} \boldsymbol{u}_{i}(\sigma)} \mathrm{d} \boldsymbol{u}_{i}(\sigma)^{\mathrm{T}}\right]\right\| \\
& <\left\|\exp \left[\int_{0}^{t}\left|\boldsymbol{u}_{i}(\sigma)\right| \mathrm{d} \boldsymbol{u}_{i}(\sigma)^{\mathrm{T}}\right]\right\| \\
& <\left\|\exp \left[B_{u_{i}} \int_{0}^{t} \mathrm{~d} \boldsymbol{u}_{i}(\sigma)^{\mathrm{T}}\right]\right\| \\
& =\left\|\exp \left\{B_{u_{i}}\left[\boldsymbol{u}_{i}(t)^{\mathrm{T}}-\boldsymbol{u}_{i}(0)^{\mathrm{T}}\right]\right\}\right\| \leqslant B_{i 1}
\end{aligned}
$$

where $B_{i 1}$ is some positive constant. For the bound of the second term in equation (42), the analysis proceeds as given below

$$
\begin{gathered}
\left\|\int_{0}^{t} \boldsymbol{\Phi}_{i}(t, \sigma) \frac{\boldsymbol{u}_{i}(\sigma)}{\boldsymbol{u}_{i}(\sigma)^{\mathrm{T}} \boldsymbol{u}_{i}(\sigma)} \dot{\tau}_{i \mathrm{~d}}(\sigma) \mathrm{d} \sigma\right\| \\
\quad<B_{i 1}\left\|\int_{0}^{t} \frac{\boldsymbol{u}_{i}(\sigma)}{\boldsymbol{u}_{i}(\sigma)^{\mathrm{T}} \boldsymbol{u}_{i}(\sigma)} \dot{\tau}_{i \mathrm{~d}}(\sigma) \mathrm{d} \sigma\right\| \\
\quad<B_{i 1}\left\|\int_{0}^{t} \boldsymbol{u}_{i}(\sigma) \dot{\tau}_{i \mathrm{~d}}(\sigma) \mathrm{d} \sigma\right\| \\
\quad<B_{i 1} B_{u_{i}}\left\|\int_{0}^{t} \dot{\tau}_{i \mathrm{~d}}(\sigma) \mathrm{d} \sigma\right\| \\
<B_{i 1} B_{u_{i}}\left\|\tau_{i \mathrm{~d}}(t)-\tau_{i \mathrm{~d}}(0)\right\| \leqslant B_{i 2}
\end{gathered}
$$

where $B_{i 2}$ is some positive constant. Since the two components of the solution in equation (42) evolve bounded, the sum of them will trivially be bounded as given in

$$
\left\|\phi_{i}(t)\right\|<B_{i 1}+B_{i 2}
$$

Note that in equation (25) it is assumed that the parameters of the flexible controller, $\phi_{i}$, are bounded. Parallel to this assumption, Theorem 2 states that once the system enters the sliding mode $s_{\mathrm{c}_{i}}=0$, the boundedness of $\phi_{i}$ is guaranteed, that is to say, equation (25) is automatically satisfied.

\subsection{Practical issues}

The analysis and the design approach presented so far have tried to illuminate the SMC problem from a theoretical perspective. In this subsection, several 
issues related to the practical applications of the discussed methodology are discussed.

\subsubsection{Chattering}

Since the control decision during the sliding mode is very dependent on the sign of a measured quantity being noisy and very close to zero, the decision on the observations along the sliding manifold exhibits sensitivity to noise. Among many alternatives available $[\mathbf{1}, \mathbf{3}, \mathbf{1 5}]$, a common approach to eliminate the chattering is to smooth the sign function, which corresponds to introducing a boundary layer [3].

In this paper, the following approximation for the $\operatorname{sgn}($.$) function is adopted$

$$
\operatorname{sgn}(x) \cong \frac{x}{|x|+\delta}
$$

where $\delta$ determines the sharpness around the origin. Since the function in equation (47) is not discontinuous at the origin, the decision mechanism softly switches to inside the boundary layer.

\subsubsection{Actuation speed}

Another important issue is the actuation speed of the system under control, i.e. the ability to respond in a timely manner to what is imposed. Since it cannot be assumed that the details concerning the dynamic model of the system are available, what causes a difficulty from a practical point of view is selection of the matrix $\xi$, which characterizes the behaviour during the reaching mode. The values of this quantity can only be set by trial-and-error due to the lack of system-specific details. Besides, if the uncertainty is large, i.e. little information about the details embodying the plant are available, $\xi$ is selected to be large, but this makes the high-frequency component of the control signal more effective compared to small $\xi$ cases. On the other hand, small $\xi$ causes slow reaching. Clearly, the selection is a matter of experience gained through the trials and knowledge about the plant dynamics.

Note that a tuning law minimizing some norm of equation (20) forces the reaching dynamics in equation (15). Uncertainties that are not taken into consideration modify this equality as $\dot{\boldsymbol{s}}_{\mathrm{p}}=$ $-\xi \operatorname{sgn}\left(s_{\mathrm{p}}\right)+\Delta$, where $\Delta$ can be seen to be the collective form of uncertainties perturbing the reaching dynamics. In this case, maintaining $\boldsymbol{s}_{\mathrm{p}}^{\mathrm{T}} \dot{\boldsymbol{s}}_{\mathrm{p}}<0$ when $\boldsymbol{s}_{\mathrm{p}} \neq \mathbf{0}$ would require $\xi_{i}>\sup \left|\Delta_{i}\right|$, where $\xi_{i}$ corresponds to the $i$ th element in the diagonal of matrix $\xi$ and $\Delta_{i}$ is the $i$ th entry of the vector $\Delta$. Clearly, this explains how the behaviour would be influenced from uncertainties that are likely to occur in practice and how the performance is affected by the selection of $\xi$.

\subsubsection{Obtaining the equivalent error from measurements}

Lastly, in this subsection, there is a focus on the construction of the $\boldsymbol{s}_{\mathrm{c}}$ of equation (20), which requires the differentiation of $s_{\mathrm{p}}$. The method adopted in this paper is to filter the measured values of $\boldsymbol{s}_{\mathrm{p}}$ and differentiate afterwards. Denote $S$ as the Laplace variable and use the linear dynamic system given as

$$
H(S)=\frac{\alpha S}{Q(S)}
$$

where $Q(0)=\alpha>0$ and $\operatorname{Real}\left\{\arg _{s}[Q(S)]=0\right\}<0$. The order of the denominator polynomial and the locations of the roots are left to the designer, because these issues require several trials to refine the selections and are subject to the application together with its operating environment.

Lastly, it should be noted that the cost of the information loss by using such a filter, whose input is $\boldsymbol{s}_{\mathrm{p}}$ and output is an estimate of $\dot{\boldsymbol{s}}_{\mathrm{p}}$, is a matter of how robust the devised control algorithm is. More explicitly, separation of the noise and the actual value of $\boldsymbol{s}_{\mathrm{p}}$ leads to a corruption in $\boldsymbol{s}_{\mathrm{p}}$, and when differentiated afterwards, some valuable information is lost together with the elimination of the noise component. Here it is assumed that the mentioned loss causes an uncertainty, which enters the system through the control channels, and which is particularly effective during the sliding mode. This uncertainty can be alleviated if it falls within the limits, allowing maintenance of the invariance during the sliding mode, as discussed above and in reference $[\mathbf{1}]$.

\section{A CASE STUDY: SMC OF A DOUBLE PENDULUM SYSTEM}

A coupled double pendulum system is used to illustrate the performance of the method discussed. The physical structure of the plant is illustrated in Fig. 2. Since the dynamics of such a mechatronic system is modelled by non-linear and coupled differential equations, precise tracking becomes a difficult objective due to the strong interdependency between the variables involved. Furthermore, the ambiguities introduced by the noise on the measured quantities make the design of a robust controller so complicated that achieving the design is a challenge 


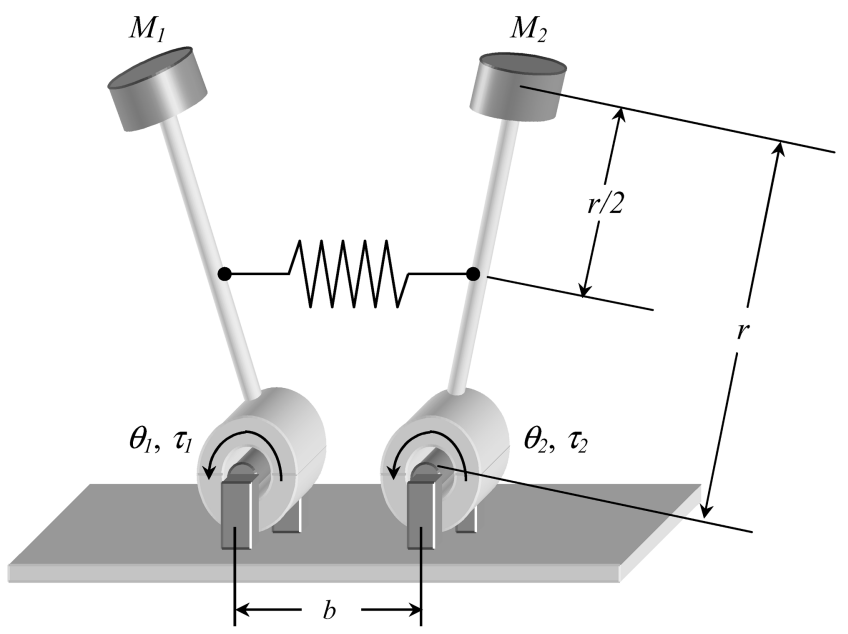

Fig. 2 Physical structure of the double pendulum system

in the conventional design framework. Therefore, for such a system, the control methodology adopted must be capable of handling the difficulties stated.

The differential equations characterizing the behaviour of the system are given by

$$
\begin{aligned}
\ddot{\theta}_{1}= & \left(\frac{M_{1} g r}{J_{1}}-\frac{k_{\mathrm{s}} r^{2}}{4 J_{1}}\right) \sin \left(\theta_{1}\right)+\frac{k_{\mathrm{s}} r}{2 J_{1}}(l-b) \\
& +\frac{\tau_{1}}{J_{1}}+\frac{k_{\mathrm{s}} r^{2}}{4 J_{1}} \sin \left(\dot{\theta}_{2}\right) \\
\ddot{\theta}_{2}= & \left(\frac{M_{2} g r}{J_{2}}-\frac{k_{\mathrm{s}} r^{2}}{4 J_{2}}\right) \sin \left(\theta_{2}\right)-\frac{k_{\mathrm{s}} r}{2 J_{2}}(l-b) \\
& +\frac{\tau_{2}}{J_{2}}+\frac{k_{\mathrm{s}} r^{2}}{4 J_{2}} \sin \left(\dot{\theta}_{1}\right)
\end{aligned}
$$

in which the angular positions and the angular velocities for each pendulum define the state vector and $g=9.81 \mathrm{~m} / \mathrm{s}^{2}$ is the gravitational acceleration constant. The control inputs, which are denoted by $\tau_{1}$ and $\tau_{2}$, are provided for the relevant pendulum by servomotors at the base. The parameters of the plant are given in Table 1 . As given in Table 1 , since $b<l$, the two pendulums repel each other in the upright

Table 1 Parameters of the double pendulum system

\begin{tabular}{lll}
\hline Mass of pendulum 1 & $M_{1}$ & $2 \mathrm{~kg}$ \\
Mass of pendulum 2 & $M_{2}$ & $2.5 \mathrm{~kg}$ \\
Moment of inertia for pendulum 1 & $J_{1}$ & $0.5 \mathrm{~kg}$ \\
Moment of inertia for pendulum 2 & $J_{2}$ & $0.625 \mathrm{~kg}$ \\
Spring constant & $k_{\mathrm{s}}$ & $100 \mathrm{~N} / \mathrm{m}$ \\
Natural length of the spring & $l$ & $0.5 \mathrm{~m}$ \\
Distance between pendulum hinges & $b$ & $0.4 \mathrm{~m}$ \\
Pendulum height & $r$ & $0.5 \mathrm{~m}$ \\
\hline
\end{tabular}

position. The model introduced in this section has been studied by Spooner and Passino [22], who discuss the decentralized adaptive control using radial basis neural networks.

In practical implementations of control structures for trajectory control of mechatronic devices, a number of difficulties are encountered, which make it difficult to achieve an accurate trajectory tracking. The simulation studies carried out address these difficulties. The first difficulty to be alleviated is the existence of the observation noise. To study the effects of this situation, which is very likely to be encountered in practice, the information used by the controller is corrupted by a noise sequence, the properties of which are given in Table 2. The second difficulty is the non-zero initial conditions for the pendulum angular positions. In order to demonstrate the reaching mode performance of the algorithm, the two pendulums are moved to arbitrarily chosen initial conditions, as given in Table 2 .

The adjustable controller parameters are set initially to zero, i.e. the parametric evolution starts from the origin. The noise sequences are Gaussian having zero mean, and the adopted filter structure is as given by

$$
H(S)=\frac{\alpha S}{Q(S)}=\frac{\alpha S}{S^{2}+2|\sqrt{\alpha}| S+\alpha}
$$

Under these conditions, in response to the reference trajectory depicted in Fig. 3, the motion observed in the phase plane is illustrated in the top row of Fig. 4, in which after a fast reaching mode, a sliding motion is enforced and is maintained by producing an appropriate control signal, which is depicted in the bottom row of Fig. 4. The smoothness of the control signals is an important result, which is a consequence of the smoothed sign function. Lastly, the

\begin{tabular}{|c|c|}
\hline Controller input vector & $\boldsymbol{u}_{i}=\left[\begin{array}{lll}e_{i} & \dot{e}_{i} & 1\end{array}\right]^{\mathrm{T}}, i=1,2$ \\
\hline Uncertainty bounds & $k_{1}=1000, k_{2}=1000$ \\
\hline Simulation stepsize & $T_{\mathrm{s}}=2.5 \mathrm{~ms}$ \\
\hline Initial errors & $\begin{array}{l}e_{1}(0)=5 \pi / 12 \mathrm{rad} \\
e_{2}(0)=-\pi / 2 \mathrm{rad} \\
\dot{e}_{1}(0)=0 \mathrm{rad} / \mathrm{s} \\
\dot{e}_{2}(0)=0 \mathrm{rad} / \mathrm{s}\end{array}$ \\
\hline Sliding line parameter & $\lambda_{1}=1, \lambda_{2}=1$ \\
\hline Noise variance & $0.33 \times 10^{-6}$ \\
\hline Noise peak value with probability $\approx 1$ & $1 \times 10^{-3}$ \\
\hline sgn function smoothing parameter & $\delta=0.25$ \\
\hline SMC design matrix & $\xi=I_{2 \times 2}$ \\
\hline Filtering parameter & $\alpha=100$ \\
\hline
\end{tabular}
evolutions in the parameters of the two controllers $\left(\phi_{i j}\right)$ are illustrated in Fig. 5, from which the bounded evolution is evident as claimed.

Table 2 Simulation data 


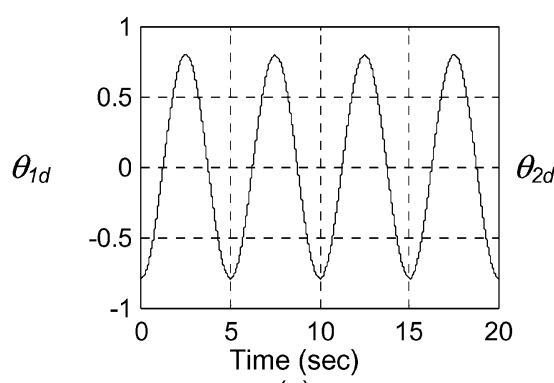

(a)

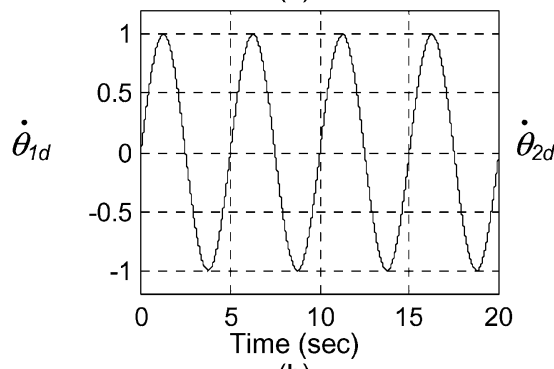

(b)

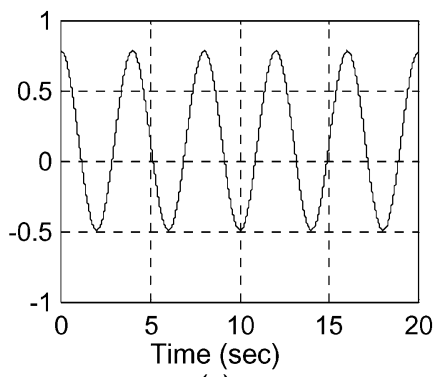

(c)

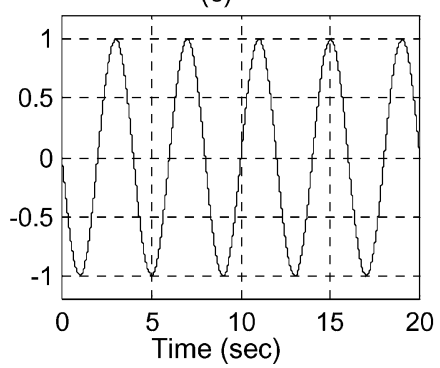

(d)

Fig. 3 Reference state trajectories

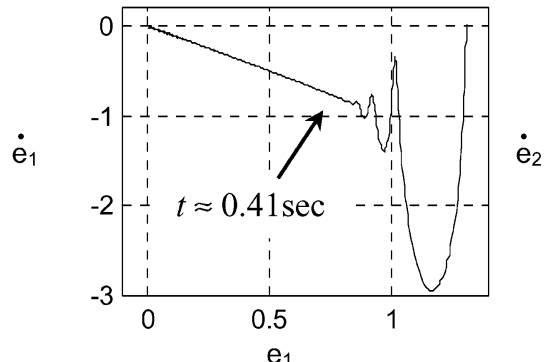

(a)

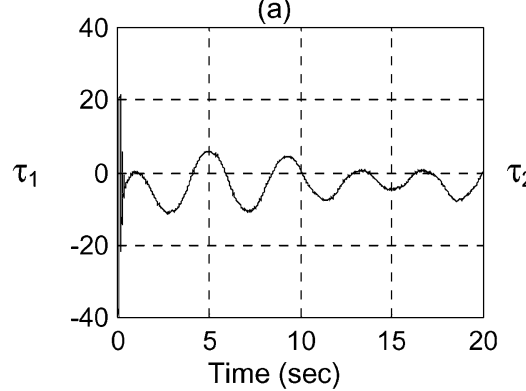

(b)
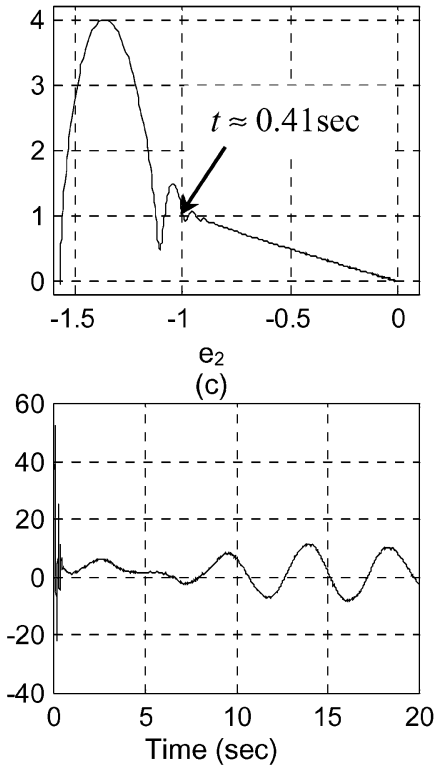

(d)

Fig. 4 Motion in the phase plane (top row) and the applied control signals (bottom row)

\section{CONCLUSIONS}

This paper discusses the design of a variable structure control strategy for a class of systems. The approach assumes that the task of SMC is achievable and the system has the structure given in equation (1). A number of issues in SMC design are discussed from the point of control engineering and an exemplar case has been studied. It is shown that the tuning strategy leads to a stable closed loop meeting the desired specifications. Furthermore, the control system retains a high degree of robustness against disturbances and uncertainties. The major contribution of the paper is the creation and maintenance of a sliding regime without knowing the analytic details describing the plant dynamics. In order to corroborate the applicability, a double pendulum system is considered. A good tracking precision is obtained without much computational effort. In Fig. 6, a bar graph composed of triplets is illustrated. The horizontal axis of the graph is the order of the plant under control and the vertical axis 


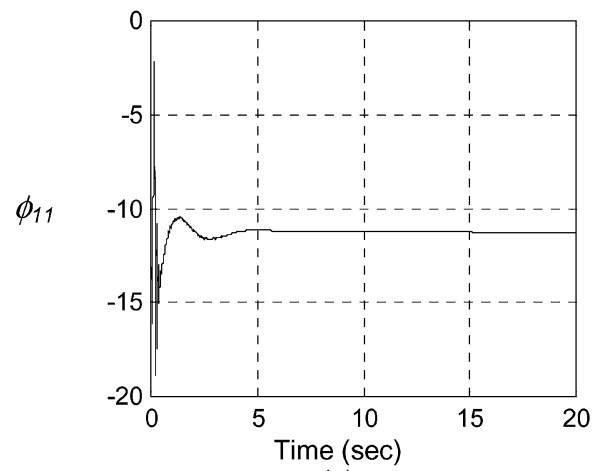

(a)

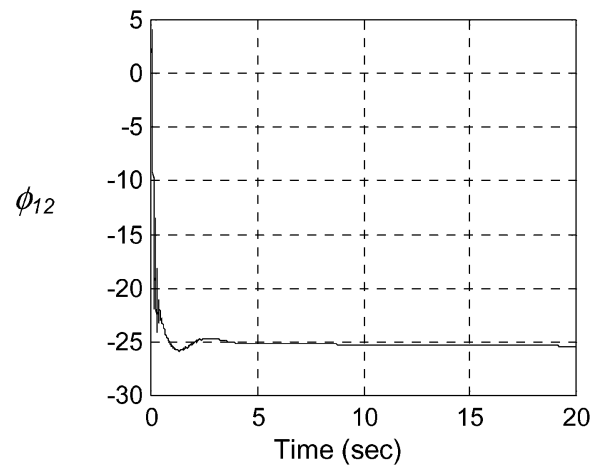

(b)

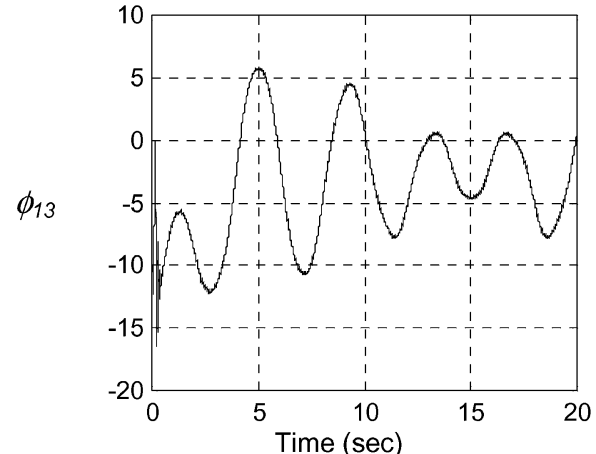

(c)

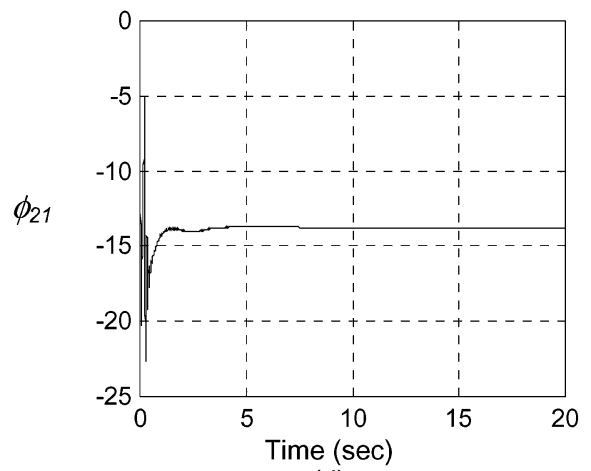

(d)

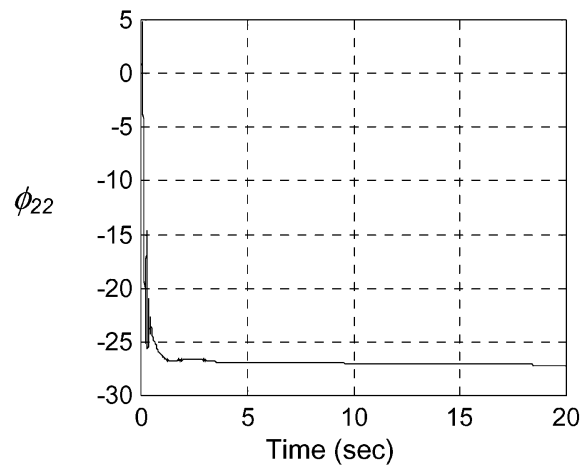

(e)

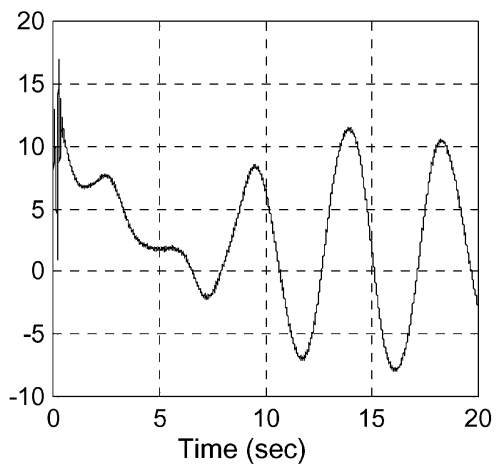

(f)

Fig. 5 Time evolution of the parameters of the two controllers

is the required number of floating-point operations (flops) to generate the control input. The leftmost component of each triplet corresponds to the required number of flops to obtain the output of the filter structure in equation (51), which is solved numerically using the fourth-order Runge-Kutta method. Since the structure adopted is the same in all four examples, the value is 79 flops. The middle component stands for the number of flops executing equations (24) and (28) once, and the rightmost component gives the sum of the first two. The values are affordable even for the average-speed microcontrollers.

Briefly, the simulation results presented demonstrate that the algorithm discussed is able to compensate for deficiencies caused by the imperfect observations of the state variables, large initial errors, and complex plant dynamics. From these points of view, the method proposed is highly promising for practical purposes. Future work aims to remove the possible drifts in the parametric evolution, thus making the uncertainty bounds adaptive.

\section{ACKNOWLEDGEMENTS}

The author thanks Professor Okyay Kaynak, Professor Xinghuo Yu, and Professor Cem Ünsal for fruitful discussions in devising the presented work. The comments and suggestions of the anonymous reviewers are gratefully acknowledged. This work was supported by TOBB Economics and Technology University, BAP Program, under Contract ETU-BAP-2006/04. 


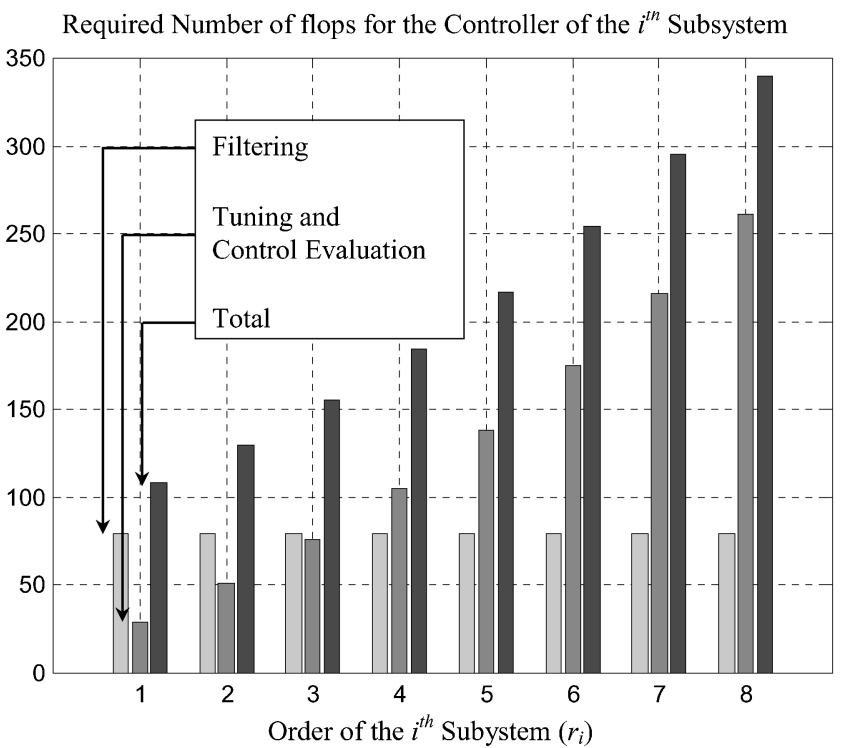

Fig. 6 Computational complexity of the algorithm

\section{REFERENCES}

1 Hung, J. Y., Gao, W., and Hung, J. C. Variable structure control: a survey. IEEE Trans. Ind. Electronics, 1993, 40, 2-22.

2 Utkin, V. I. Sliding modes in control optimization, 1992 (Springer-Verlag, New York).

3 Slotine, J.-J. E. and Li, W. Applied nonlinear control, 1991 (Prentice-Hall, Englewood Cliffs New Jersey).

4 Knapp, T. D., Budman, H. M., and Broderick, G. Adaptive control of a CSTR with neural network model. J. Process Control, 2001, 11, 53-68.

5 Camacho, O. and Rojas, R. A general sliding mode controller for nonlinear chemical processes. Trans. ASME, J. Dynamic Systems, Measmt, and Control, 2000, 122, 650-655.

6 Hanczyc, E. and Palazoglu, A. Sliding mode control of nonlinear distributed parameter chemical processes. Ind. Engng Chem. Res., 1995, 34, 557-566.

7 Colantonio, M. C., Desages, A. C., Romagnoli, J. A., and Palazoglu, A. Nonlinear control of a CSTR: disturbance rejection using sliding mode control. Ind. Engng Chem. Res., 1995, 34, 2383-2392.

8 Poznyak, A. S., Yu, W., and Sanchez, E. N. Identification and control of unknown chaotic systems via dynamic neural networks. IEEE Trans. Circuits and Systems - I: Fundamental Theory and Applications, 1999, 46, 1491-1495.

9 Young, K. D., Utkin, V. I., and Özgüner, Ü. A control engineer's guide to sliding mode control. IEEE Trans. Control Systems Technol., 1999, 7, 328-342.
10 Kaynak, O., Harashima, F., and Hashimoto, H. Variable structure systems theory, as applied to subtime optimal position control with an invariant trajectory. Trans. IEE Japan, 1984, Sec. E, 104, 47-52.

11 Misawa, E. A. Discrete-time sliding mode control: the linear case. Trans. ASME, J. Dynamic Systems, Measmt, and Control, 1997, 119, 819-821.

12 Misawa, E. A. Discrete-time sliding mode control for nonlinear systems with unmatched uncertainties and uncertain control vector. Trans. ASME, J. Dynamic Systems, Measmt, and Control, 1997, 119, 503-512.

13 Sabanovic, A., Sabanovic, N., Jezernik, K., and Wada, K. Chattering free sliding modes. In Proceedings of the Third Workshop on Variable structure systems and Lyapunov design, Naples, Italy, 1994, pp. 143-148.

14 Bartolini, G., Ferrera, A., Usai, E., and Utkin, V. I. On multi-input chattering-free second-order sliding mode control. IEEE Trans. Autom. Control, 2000, 45, 1711-1717.

15 Erbatur, K., Kaynak, O., and Sabanovic, A. A study on robustness property of sliding mode controllers: a novel design and experimental investigations. IEEE Trans. Ind. Electronics, 1999, 46, 1012-1018.

16 Sira-Ramirez, H. and Colina-Morles, E. A sliding mode strategy for adaptive learning in adalines. IEEE Trans. Circuits and Systems - I: Fundamental Theory and Applics, 1995, 42, 1001-1012.

17 Yu, X., Zhihong, M., and Rahman, S. M. M. Adaptive sliding mode approach for learning in a feedforward neural network. Neural Computing Applics, 1998, 7, 289-294.

18 Efe, M. Ö., Kaynak, O., and Yu, X. Sliding mode control of a three degrees of freedom anthropoid robot by driving the controller parameters to an equivalent regime. Trans. ASME, J. Dynamic Systems, Measmt and Control, 2000, 122, 632-640.

19 Parma, G. G., Menezes, B. R., and Braga, A. P. Sliding mode algorithm for training multilayer artificial neural networks. Electronics Lett., 1998, 34, 97-98.

20 Edwards, C. and Spurgeon, S. K. Sliding mode control - theory and applications, 1998 (Taylor and Francis, London).

21 Denker, A. and Kaynak, O. Applications of VSC in motion control systems. In Variable structure and Lyapunov control (Eds A. S. I. Zinober), 1994 (Springer-Verlag, London).

22 Spooner, J. T. and Passino, K. M. Decentralized adaptive control of nonlinear systems using radial basis neural networks. IEEE Trans. Autom. Control, 1999, 44, 2050-2057. 